\title{
Estetrol: ¿un nuevo paradigma en terapia ginecológica?
}

\author{
Estetrol: a new paradigm in gynecological therapy? \\ Teresa Navarrete Horta, ${ }^{*}$ Francisco Javier Guerrero Carreño, ${ }^{\ddagger}$ \\ Paulina Valdés Aguerrebere, ${ }^{\S}$ Leopoldo Alejandro Vázquez Estrada* \\ Citar como: Navarrete HT, Guerrero CFJ, Valdés AP, Vázquez ELA. Estetrol: zun nuevo paradigma en terapia \\ ginecológica? Acta Med GA. 2022; 20 (1): 50-57. https://dx.doi.org/10.35366/103556
}

\section{Resumen}

El estetrol (E4) es un estrógeno natural producido por el feto humano y que pasa a la sangre materna en niveles relativamente altos durante el embarazo. A lo largo de las últimas dos décadas, se ha demostrado que sus perfiles farmacodinámico y farmacocinético, su tolerabilidad y su margen de seguridad son muy favorables. En este sentido, E4 potencialmente representa un gran avance en la terapia ginecológica para la anticoncepción y el manejo de la menopausia; también muestra aplicabilidad en otros campos terapéuticos como: cánceres hormono-dependientes (mama y próstata), osteoporosis, neuroprotección, dolor muscular y cicatrización de heridas. Presentamos una revisión de la evidencia actual de esta "nueva" opción terapéutica hormonal cuya inclusión a la práctica clínica ginecológica parece inminente.

Palabras clave: Estetrol, E4, anticoncepción, menopausia, terapia hormonal.

\section{INTRODUCCIÓN}

Hace más de 50 años, el grupo de investigadores del Instituto Karolinska en Estocolmo, Suecia, liderados por Egon Diczfalusy, comenzó a investigar el complejo sistema de intercambios hormonales que tiene lugar entre la madre y el feto a través de la placenta. ${ }^{1}$ En los siguientes 20 años desarrollaron un nuevo concepto: la existencia durante el embarazo de una unidad funcional compuesta por un órgano esteroidogénico incompleto (la placenta), interpuesto entre un sistema metabólico esteroidogénico completo (el organismo materno) y un segundo sistema incompleto (el feto). Este último tenía la característica única de ser capaz

\section{Abstract}

Estetrol (E4) is a natural estrogen produced by the human fetus and passes into the maternal blood at relatively high levels during pregnancy. Over the past two decades, its pharmacodynamic and pharmacokinetic profiles, tolerability, and safety margin have been shown to be highly favorable. In this sense, E4 potentially represents a great advance in gynecological therapy such as contraception and the management of menopause. E4 also shows applicability in other therapeutic fields such as hormone-dependent cancers (breast and prostate), osteoporosis, neuroprotection, muscle pain, and wound healing. We present hereinafter the current evidence on this "new" hormonal therapy option whose inclusion in gynecological clinical practice seems imminent.

Keywords: Estetrol, E4, contraception, menopause, hormonal therapy.

de compensar las deficiencias enzimáticas de la placenta. Específicamente con relación a la síntesis de esteroides estrogénicos de la unidad feto-placentaria, demostraron que el estetrol (15 $\alpha$-hidroxiestradiol) es sintetizado a partir de estradiol (E2) y de estriol (E3) exclusivamente por el hígado fetal durante el embarazo humano y llega a la circulación materna a través de la placenta (Figura 1). El estetrol (E4) es una hormona esteroidea con cuatro grupos hidroxilos $(-\mathrm{OH})$, dos más que el estradiol (E2), el estrógeno fisiológicamente producido por las células de la granulosa de los ovarios humanos (Figura 2). El hígado fetal ${ }^{2}$ es el sitio exclusivo responsable de $15 \alpha-$ y $16 \alpha$-hidroxilación. Por esta razón, E4 sólo está presente durante el embarazo desde

\footnotetext{
* Ginecobstetra, Biología de la Reproducción Humana. Hospital Angeles Lindavista. México.

‡ Ginecobstetra, Perinatólogo. Hospital Angeles Lindavista. México.

$\S$ Ginecobstetra, Hospital Médica Sur. México.
}

\section{Correspondencia:}

Leopoldo Alejandro Vázquez Estrada

Correo electrónico: Ipldvzqz@gmail.com

www.medigraphic.com/actamedica

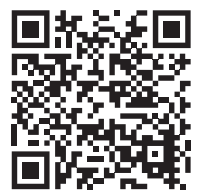


las nueve semanas de gestación hasta poco después del nacimiento. ${ }^{3}$ Los dos hidroxilos $(-\mathrm{OH})$ adicionales tienen un impacto crucial en la farmacocinética oral: la vida media de E4 es de 20 a 28 horas, en comparación con sólo 10-20 minutos para estriol (E3), 1-2 horas para E2 natural y 10-12 horas para E2 micronizado E2. ${ }^{4}$ El estetrol (E4) se metaboliza mínimamente, y no se reconvierte en estriol (E3) o estradiol (E2). La detección de E4 en la orina materna tiene lugar a partir de las nueve semanas de embarazo, con concentraciones de E4 no conjugado en constante aumento a aproximadamente $1 \mathrm{ng} / \mathrm{mL}$ ( $>3 \mathrm{nmol} / \mathrm{L}$ ) hacia el final del embarazo. ${ }^{3}$ Su síntesis hepática disminuye durante la infancia y es baja, ausente o indetectable en los adultos. Aunque su acción fisiológica no se conoce con certeza, el estetrol parece contribuir a los efectos de los estrógenos que tienen lugar durante el embarazo, ya que sus niveles aumentan constantemente con el avance de la gestación y los niveles fetales son más altos que los maternos. Como hormona de origen exclusivamente fetal, el descubrimiento de E4 generó un gran entusiasmo con respecto a su potencial como índice para identificar y estudiar embarazos complicados. En consecuencia, se investigó $^{5-9}$ la correlación entre los niveles circulantes de E4 en el plasma materno durante diversas alteraciones del embarazo (isoinmunización Rh, diabetes mellitus, colestasis intrahepática, preeclampsia y muerte fetal intrauterina). Los resultados de estos estudios revelaron que, debido a la gran variación intra e interindividual de sus niveles plasmáticos, E4 tenía un valor limitado en el seguimiento

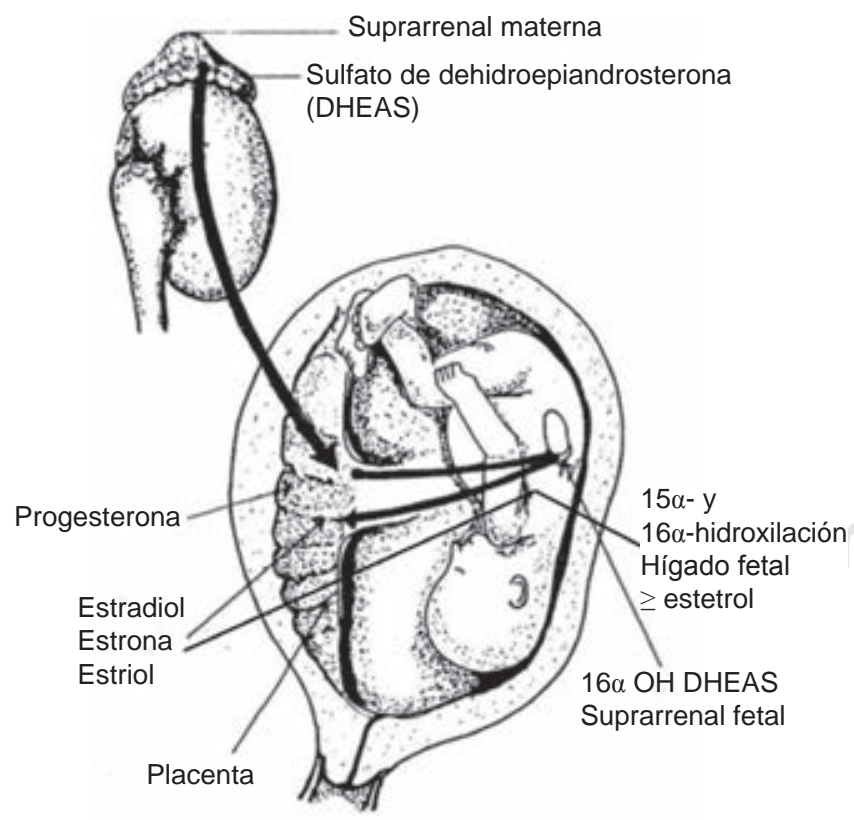

Figura 1: Esteroidogénesis en la unidad fetoplacentaria.

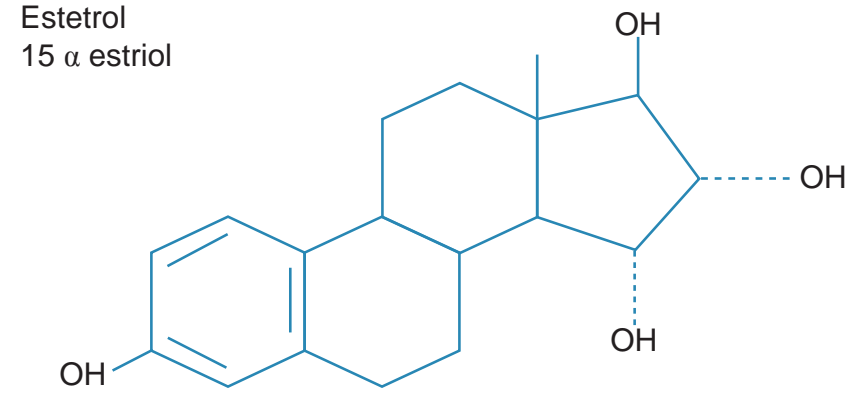

Figura 2: Estructura molecular del estetrol (E4) $5 \alpha$-hidroxiestradiol; Estra-1,3,5(10)-triene-3,15 $\alpha, 16 \alpha, 17 \beta$-tetrol.

del embarazo y no resultó un marcador específico para la identificación de anomalías fetales. Asimismo, se concluyó que el E4 tenía una acción biológica débil con base en su baja afinidad de unión a los receptores estrogénicos (RE), desde $0.3 \%$ (rata) ${ }^{10}$ a $6.25 \%$ (humano), ${ }^{11}$ y sólo 2 a $3 \%$ de potencia comparado con estradiol (E2) en una serie de ensayos in vitro e in vivo. ${ }^{12-14}$ Debido a ello, después de 20 años de trabajo experimental, la investigación de E4 fue prácticamente abandonada.

En los últimos años, con base en nuevos datos, se ha experimentado un renacimiento de las actividades de investigación preclínica y clínica con el objetivo de explorar el potencial de E4 para su uso terapéutico en humanos, ya que la exposición a este esteroide estrogénico en cantidades significativas, tanto en fetos masculinos como femeninos, demuestra un alto perfil de seguridad y debido a ello probablemente representaría una excelente opción terapéutica. En consecuencia, en 2001 se inició un proyecto liderado por el profesor Herjan Coelingh Bennink en los Países Bajos para investigar las propiedades farmacológicas de E4 con tecnologías de última generación. ${ }^{15}$

\section{SÍNTESIS Y PROPIEDADES FARMACOLÓGICAS DE ESTETROL (E4)}

El proceso fármaco-químico de síntesis de E4 a partir de estrona (E1) da como resultado la producción de E4 de muy alta pureza (>98\%) sin contaminación con estradiol (E2), ${ }^{16}$ propiedades muy favorables para el desarrollo de un producto hormonal con fines terapéuticos. La nueva investigación ha revelado que E4 se une selectivamente a ambos receptores de estrógeno (RE) ${ }^{17}$ con mayor preferencia por el RE $\alpha$ y menor a RE $\beta$. No se observa toxicidad en ratas, con dosis máximas de $10 \mathrm{mg} / \mathrm{kg} /$ día durante cuatro semanas, ${ }^{18}$ ni tampoco en humanos, con una dosis máxima de $100 \mathrm{mg} .{ }^{4}$ Debido a sus propiedades farmacológicas ${ }^{16}$ y metabólicas, ${ }^{2,4,19}$ especialmente su alta biodisponibilidad 
oral relacionada con la dosis, ${ }^{4,19}$ la ausencia de unión a $\mathrm{SHBG}^{20}$ y vida media de eliminación de 28 horas, puede ser usado por vía oral una vez al día.

En estudios preclínicos, E4 se comporta como un agonista de estrógenos en todos los tejidos investigados: hueso, ${ }^{19}$ vagina, miometrio y endometrio ${ }^{21}$ e hipotálamo (inhibición de bochornos ${ }^{22}$ e inhibición de la ovulación). ${ }^{23}$ Paradójicamente, este esteroide actúa como un antagonista de estrógenos en tejido tumoral de mama en presencia de E2. ${ }^{18}$ Debido a su perfil farmacológico, el estetrol (E4) puede ser clasificado como un "SERM fetal humano natural"15 o más recientemente denominado estrógeno natural con acción selectiva en tejidos (NEST ${ }^{\circledR}$ por sus siglas en inglés). ${ }^{24}$ La interacción de estetrol (E4) con la función hepática en modelos in vitro demuestra primero: un metabolismo "lento",${ }^{17}$ lo que explica su larga vida media; segundo: ausencia de inhibición del citocromo P450, lo que puede implicar una menor probabilidad de interacción farmacológica; y tercero: no estimula la síntesis de $\mathrm{SHBG},{ }^{20}$ lo que sugiere un riesgo potencialmente menor de tromboembolismo venoso que es un efecto secundario de todos los estrógenos y de los moduladores selectivos de los receptores estrogénicos (SERM) sintéticos.

Además, E4 muestra ser un estrógeno con actividad biológica más potente de lo que se estimaba. Aunque en los estudios farmacológicos el estetrol fue 10-20 veces menos potente en comparación con el etinilestradiol, las dosis únicas de $10 \mathrm{mg}$ de E4 son capaces de suprimir eficazmente la secreción de hormona luteinizante $(\mathrm{LH})$ y hormona folículo-estimulante (FSH) en mujeres postmenopáusicas. ${ }^{25}$ La diferencia puede explicarse porque, en su momento, era escaso el conocimiento de las propiedades metabólicas de E4, debido a que la mayoría de los estudios anteriores fueron realizados in vitro y muy pocos in vivo. En la actualidad, los estudios realizados de manera más adecuada de absorción, distribución, metabolismo y excreción (ADME) demuestran una farmacocinética (Tabla 1) del E4 más favorable. ${ }^{26}$

\section{ESTETROL EN ANTICONCEPCIÓN}

Los anticonceptivos orales combinados (AOC) tradicionalmente contienen un estrógeno y una progestina. La inhibición de la ovulación es la acción preponderante de la progestina, mientras que los estrógenos estabilizan el endometrio, regulan el sangrado vaginal y reducen el desarrollo folicular inhibiendo la secreción de hormona estimulante del folículo (FSH). El etinilestradiol (EE) es el estrógeno más utilizado debido a su eficacia y seguridad demostradas a lo largo de los últimos 60 años, ya que se asocia con un patrón de sangrado regular. Sin embargo, su impacto en la función hepática y el endotelio vascular puede aumentar el riesgo de desarrollar complicaciones cardiovasculares trombóticas (arteriales y venosas) durante el uso de AOC. Se han desarrollado diferentes estrategias para reducir este efecto: disminuyendo la dosis de $\mathrm{EE}$, mejorando la modulación hormonal con progestágenos androgénicos y, desde 2009, sustituyendo EE con E2; ${ }^{27}$ sin embargo, la combinación óptima está todavía por descubrirse. La introducción de E4 puede mejorar la seguridad y tolerabilidad de los AOC. Hay razones para asumir que E4 sea seguro como componente de los AOC, ya que a diferencia de $\mathrm{EE}$, E4 es un esteroide natural presente durante el embarazo humano en niveles crecientes. ${ }^{28} \mathrm{~A}$ término, el feto sintetiza alrededor de $3 \mathrm{mg}$ de E4 por día ${ }^{29}$ y la exposición fetal diaria es comparable al tratamiento de mujeres adultas con una dosis oral de 50 a $55 \mathrm{mg}$ de E4 por día. ${ }^{4}$ Ya que esta alta exposición muestra ser segura para el feto, esto podría extrapolarse a un adecuado perfil de seguridad, incluso a dosis altas en la terapéutica ginecológica. Por otra

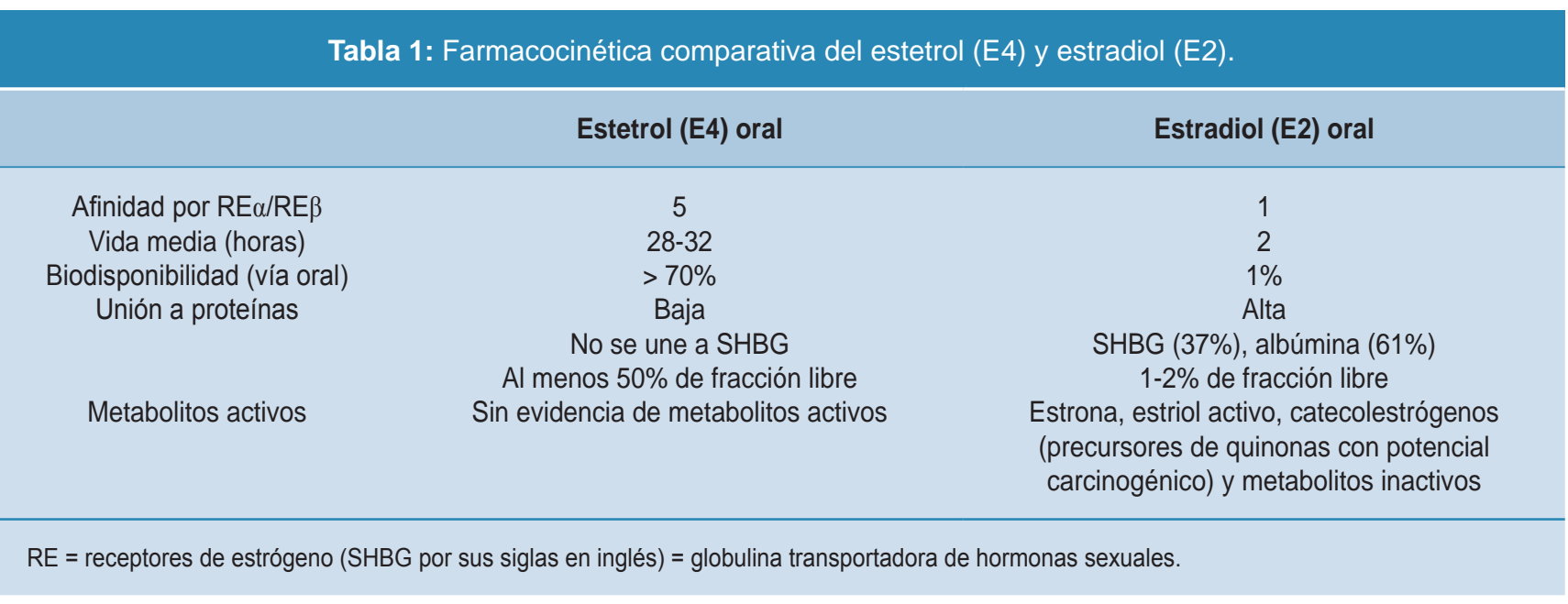


parte, E4 tiene afinidad exclusiva por los receptores RE- $\alpha$ y RE- $\beta$ y ninguna para otro receptor de esteroides ni de otros 124 fármacos. ${ }^{17}$ En humanos, dosis de E4 de hasta $40 \mathrm{mg} /$ día durante 28 días en mujeres postmenopáusicas no han mostrado ningún efecto secundario importante y una mínima interacción con la función hepática. ${ }^{25}$

\section{INHIBICIÓN DE LA OVULACIÓN}

Los datos preclínicos muestran que el E4 es capaz de inhibir la ovulación como agente único. ${ }^{23}$ El efecto anovulatorio de E4 depende de la dosis y su potencia resultó ser unas 18 veces menor que la de EE. En un estudio clínico fase II, ${ }^{30}$ se evaluó la inhibición de la ovulación con diferentes combinaciones de E4 (5 y $10 \mathrm{mg}$ ) con drospirenona (DRSP) de $3 \mathrm{mg}$. E4 (5, 10 o $20 \mathrm{mg})$ con levonorgestrel (LNG) de $150 \mu \mathrm{g}$ empleando como comparador el AOC DRSP $3 \mathrm{mg} /$ etinilestradiol $20 \mu$ g; todos en esquema 24/4. No se detectó ovulación en ninguno de los grupos de tratamiento. La inhibición de la actividad folicular fue proporcional a la dosis de E4, la mayor supresión se observó con dosis de 20 mg de E4 equivalente a la dosis de $20 \mu \mathrm{g}$ de etinilestradiol. El estudio FIESTA, ${ }^{31}$ diseñado para encontrar la dosis de E4, así como los patrones de sangrado de la combinación estetrol 15 o 20 mg combinado con DRSP ( $3 \mathrm{mg})$ o LNG (150 $\mu \mathrm{g})$ en régimen 24/4, empleando la combinación dienogest (DNG)/valerato de estradiol (ValE2) como comparador, demuestra que de los cuatro regímenes estudiados, la combinación de E4 $15 \mathrm{mg} /$ DRSP 3 mg tuvo el mejor desempeño en el control del ciclo: para el ciclo 6, la incidencia de sangrado/manchado intercíclico y ausencia de sangrado por deprivación, fueron más bajas en el grupo de $15 \mathrm{mg}$ de E4/DRSP 3 mg (33.8 y 3.5\%, respectivamente). En el grupo de referencia ValE2/DNG, estas proporciones fueron 47.8 y $27.1 \%$, respectivamente.

Asimismo, como objetivos secundarios, se evaluaron la aceptabilidad y la satisfacción de las usuarias, ${ }^{32}$ mostrando que las combinaciones E4/DRSP tienen un mayor efecto en la proporción de usuarias satisfechas con su régimen (73.1\%) que las combinaciones E4/LNG (50.6\%). Además, la combinación de E4 15 mg/DRSP 3 mg tuvo un efecto más favorable en el peso corporal (36.7\% reportaron disminución $\geq 2 \mathrm{~kg}$ a los seis meses de uso).

Con el objetivo de evaluar la eficacia anticonceptiva de esta combinación, se realizó el estudio fase III (FREEDOM Female Response Concerning Efficacy and Safety of Estetrol/ Drospirenone as Oral Contraceptive in a Multicentric Study). El estudio se ha llevado a cabo en más de 3,000 mujeres en Estados Unidos, Canadá, Europa y Rusia. Al momento de escribir este artículo, la información de ClinicalTrials.gov (NCT02817828 y NCT02817841) revela que el estudio ha concluido, aunque no se han publicado resultados. Al parecer su eficacia y patrón de seguridad han sido muy favorables, por lo que se espera que durante 2021 sea sometido para su aprobación ante la Agencia Europea del Medicamento y la FDA.

\section{EFECTOS ENDOCRINOS Y METABÓLICOS DEL ANTICONCEPTIVO CON E4 15 MG/DRSP 3 MG}

El AOC óptimo, además de ser capaz de bloquear eficazmente la ovulación, debe provocar cambios mínimos en la hemostasia, metabolismo de los lípidos y función hepática. La farmacodinamia del AOC E4/DRSP fue investigada en mujeres sanas en un estudio de búsqueda de dosis, ${ }^{33}$ demostrándose que los niveles de SHBG (globulina transportadora de hormonas sexuales) fueron mínimamente afectados en las usuarias de las combinaciones E4/DRSP y E4/LNG, en comparación con las usuarias de EE/DRSP en quienes se observó un aumento significativo. Del mismo modo, se observaron menores efectos sobre las lipoproteínas y triglicéridos, siendo significativamente más bajos que los del grupo EE/DRSP. Además, no se observó impacto alguno en los marcadores óseos, ni alteraciones en los niveles de factores insulinoides (IGF-I, GH). Un estudio más reciente ${ }^{34}$ confirma que el tratamiento con E4/DRSP tiene efectos mínimos sobre los parámetros endocrinos y metabólicos. Los efectos sobre las gonadotropinas, cortisol, globulina transportadora de corticoesteroides (CBG), angiotensinógeno, SHBG y triglicéridos fueron menos pronunciados en comparación con los productos que contienen EE. Aunque son necesarios estudios adicionales para establecer el perfil de seguridad de este nuevo AOC, los resultados preliminares del estudio fase III FREEDOM (resultados pendientes de publicación) parecen delinear un adecuado perfil de seguridad.

Aunque el tromboembolismo venoso (TEV) es un evento poco común entre las mujeres sanas en edad reproductiva (incidencia de cinco a 10 eventos por 10,000 mujeresaño), el uso de anticonceptivos orales combinados (AOC) puede aumentar el riesgo de TEV, en comparación con las no usuarias. Un metaanálisis reciente ${ }^{35}$ muestra que los AOC que contienen determinadas progestinas (ciproterona, desogestrel, dienogest, drospirenona, gestodeno, norgestimato) podrían conferir un mayor riesgo de TEV en comparación con los AOC que contienen levonorgestrel. Este hallazgo debe considerarse en el contexto del riesgo general de TEV entre las mujeres en edad reproductiva. Cualquier pequeño aumento en el riesgo relativo explica una pequeña cantidad de eventos a nivel de población. Suponiendo un riesgo de 9-10 eventos de TEV por 10,000 mujeres-año entre las mujeres que usan $\mathrm{AOC}$ que contienen levonorgestrel (LNG), las mujeres que usan AOC con otras progestinas pueden tener un riesgo 1.5 a 2.0 veces mayor, lo que resulta en un riesgo absoluto de aproxima- 
damente 14-20 eventos de TEV por 10,000 mujeres-año, o 5-10 eventos adicionales por 10,000 mujeres-año. Estos estudios muestran la experiencia de las combinaciones de las diferentes progestinas combinadas con EE. Sin embargo, el uso de la progestina sola como anticonceptivo oral parece no incrementar el riesgo de TEV. ${ }^{36,37} \mathrm{Al}$ ser E4 un estrógeno natural menos potente que $\mathrm{EE}$, la posibilidad de que la combinación anticonceptiva E4/DRSP tuviese menor riesgo de TEV fue investigada evaluando su impacto sobre las variables hemostáticas asociadas con incremento de riesgo de TEV. ${ }^{38,39}$ En estos estudios, los cambios en los parámetros de hemostasia (fibrinógeno, protrombina, factor VII, factor VIII, factor de von Willebrand, antitrombina, actividad proteína $S$, proteína $S$ libre, proteína C, inhibidor de la vía del factor tisular (TFPI) libre, plasminógeno, inhibidor del activador del plasminógeno tipo 1 (PAI-1), activador de plasminógeno tisular (tPA), resistencia a la proteína C activada (APCr) basado en potencial de trombina endógena (ETP) (expresado como nAPCsr), dímero D y fragmento de protrombina $1+2$ y concentración de SHBG) fueron determinados previamente y después de tres y seis meses de tratamiento empleando E4 $15 \mathrm{mg} /$ DRSP $3 \mathrm{mg}$ y los AOC: LNG 150 mg/EE $30 \mu \mathrm{g}$, DRSP 3 mg/EE $20 \mu \mathrm{g}$ como comparadores. Después del tratamiento con seis ciclos de E4/DRSP los cambios en los parámetros de hemostasia fueron menores o similares a los observados para EE/LNG. Estos cambios fueron similares, pero más pronunciados con EE/DRSP, lo que apoya la hipótesis de que el efecto de los AOC sobre la hemostasia está mediado principalmente por el componente estrogénico. Se concluye que la combinación E4/DRSP muestra tener un menor efecto procoagulante. Sin embargo, se necesitan estudios amplios para proporcionar más información sobre el riesgo tromboembólico venoso de E4/DRSP.

\section{ESTETROL EN TERAPIA HORMONAL DE LA MENOPAUSIA}

Debido a los potenciales riesgos de seguridad relacionados con las terapias hormonales percibidos tanto por médicos como por pacientes, muchas mujeres continúan evitando el tratamiento para los síntomas de la menopausia. Los estudios también subrayan la necesidad no satisfecha en la actualidad de un estrógeno con un mejor perfil riesgo/ beneficio. Las investigaciones preclínicas y clínicas hasta la fecha indican que E4 podría solucionar esta necesidad insatisfecha, ya que puede ser útil especialmente para el tratamiento de la sintomatología vasomotora, la atrofia vaginal, así como en la prevención y tratamiento de la osteoporosis.

Los estudios preclínicos ${ }^{19,21}$ revelaron que E4 actúa como un estrógeno en la vagina, el útero y los huesos.
En un modelo experimental de inestabilidad vasomotora en ratas, E4 suprimió el aumento de la temperatura de la piel de la cola inducida por naloxona. ${ }^{22}$ En el estudio de farmacodinamia clínico, ${ }^{25}$ el tratamiento con E4 comprobó los efectos estrogénicos dependientes de la dosis sobre el sistema endocrino, marcadores de recambio óseo y lípidos y lipoproteínas. El efecto sobre los triglicéridos fue mínimo al igual que los efectos sobre las variables hemostáticas. Cuantitativamente, los efectos de E4 10 mg fueron similares a $2 \mathrm{mg}$ de valerato de estradiol.

Con el fin de encontrar la dosis más adecuada para su empleo clínico, se llevó a cabo un estudio abierto de dosis múltiples ${ }^{40}$ en 49 mujeres postmenopáusicas. Veinte mujeres con útero intacto fueron asignadas al azar para recibir 2 mg de E4 $(n=10)$ o 2 mg de valerato de estradiol (ValE2) $(n=10)$ durante 28 días. En forma abierta fueron asignadas 10 mujeres (con útero intacto $y \geq 35$ sofocos/semana) a recibir 10 mg de E4; y de las 19 mujeres histerectomizadas se asignaron 10 a recibir $20 \mathrm{mg}$ y nueve la dosis de $40 \mathrm{mg}$ de E4 durante 28 días. Los resultados mostraron que no se presentaron eventos adversos graves relacionados con el tratamiento. En todos los grupos hubo un cambio de células vaginales parabasales a superficiales, lo que indica un efecto estrogénico potencial para el tratamiento de la atrofia vulvovaginal. El grosor del endometrio se mantuvo estable en el grupo de 2 mg de E4, el cual aumentó con 2 mg de ValE2 y con 10 mg de E4. Se observó una disminución en el número medio de sofocos y sudoración con 2 y $10 \mathrm{mg}$ de E4 y 2 mg de ValE2. Se concluye que E4 en un rango de dosis de 2 a $40 \mathrm{mg}$ por día mejoró la citología vaginal y los síntomas vasomotores en mujeres postmenopáusicas; E4 produjo proliferación endometrial con la dosis de $10 \mathrm{mg}$.

El estudio multicéntrico (fase II) para identificar la dosis mínima eficaz de E4 para la remisión de la sintomatología vasomotora $^{41}$ incluyó 257 mujeres postmenopáusicas (32 histerectomizadas), sintomáticas con $\geq 7$ bochornos moderados a severos por día o 50 por semana, asignadas aleatoriamente a recibir 2.5, 5, 10 o 15 mg de E4 o placebo diariamente VO (vía oral) por un periodo de 12 semanas. Los resultados muestran que todas las dosis de E4 fueron capaces de disminuir la frecuencia e intensidad de los bochornos; sin embargo, sólo la dosis de 15 mg de E4 tuvo una eficacia estadísticamente significativa versus placebo, por lo que fue considerada la dosis terapéutica mínima eficaz. Su perfil de seguridad aparentemente favorable deberá confirmarse en los ensayos fase III (estudio I en Europa, Rusia y Sudamérica; estudio II en Norteamérica), ya en fase de reclutamiento (ClínicalTrials.gov NCT04209543). Las dosis de 15 y 20 mg se compararán con placebo durante un año en grandes cohortes de mujeres postmenopáusicas ( $n=1,200$ en el estudio I; $n=1,000$ en el estudio II). Lamentablemente se detuvieron en abril de 2020 debido 
a la pandemia, pero se espera continuar una vez se restablezcan las condiciones adecuadas.

Una característica farmacológica de E4 particularmente atractiva para su uso en terapia hormonal se debe a su interacción con los receptores estrogénicos (RE). Los estudios preclínicos indican que E4 presenta un perfil biológico similar a los moduladores selectivos del receptor de estrógeno (SERM). Se ha descrito que el estetrol (E4) es un estrógeno con perfil distintivo de la activación del receptor de estrógeno alfa $(R E \alpha)$, ya que E4 activa el RE $\alpha$ nuclear, pero es un antagonista del RE $\alpha$ de la membrana. ${ }^{42}$ Este perfil original de activación del RE $\alpha$ es único y caracteriza a E4 como SERM "natural". ${ }^{43}$ En un estudio preclínico ${ }^{44}$ se demuestra que E4 actúa como un estrógeno débil al estimular células de cáncer de mama dependiente de somatotropina $(\mathrm{GH})$ sólo en concentraciones superiores a las que han sido empleadas en terapia de la menopausia. Además, E4 presenta una actividad antitumoral al disminuir el potente efecto proliferativo del estradiol (E2). Si bien, el receptor de estrógeno alfa (RE $\alpha)$ es el receptor predominante que media los efectos de E4, su modulación estrogénica débil/ antiestrogénica resulta de la activación diferencial de estas vías de señalización: agonista en el receptor nuclear (efectos beneficiosos sobre hueso, vagina, endometrio y sistema cardiovascular), antagonista del receptor de la membrana (bloquea la angiogénesis tumoral), efectos antagonistas y agonistas mixtos: en el hígado y la mama.

En modelos in vitro, E4 antagonizó el efecto proliferativo tumoral de E2 en células de cáncer de mama (MCF-7 y LTED). ${ }^{45}$ En un modelo in vivo en ratas con tumores de mama inducidos químicamente, el E4 a dosis altas previno por completo el desarrollo de tumores de mama en forma comparable a la ovariectomía. Asimismo, E4 inhibió el crecimiento de tumores mamarios preexistentes. ${ }^{46}$ El primer estudio clínico prospectivo, aleatorizado controlado con placebo ${ }^{47}$ en 30 mujeres pre y postmenopáusicas con cáncer de mama temprano con receptor de estrógeno positivo investigó el efecto de 14 días de tratamiento preoperatorio con $20 \mathrm{mg}$ de E4 por día sobre los marcadores de proliferación tumoral, la expresión del receptor de esteroides sexuales y los parámetros endocrinos. Se observó que E4 tuvo un efecto proapoptótico significativo en el tejido tumoral, mientras que la expresión de Ki67 se mantuvo sin cambios tanto en mujeres premenopáusicas como postmenopáusicas. E4 aumentó la concentración de globulina transportadora de hormonas sexuales, reduciendo significativamente las concentraciones de estradiol biodisponibles. Los niveles de hormona estimulante del folículo disminuyeron sólo en mujeres postmenopáusicas. Los niveles sistémicos del factor de crecimiento de insulina tipo 1 disminuyeron significativamente, al igual que la expresión del RE $\alpha$ epitelial intratumoral y se encontró una tendencia hacia un aumento de la expresión del RE $\beta$. Estos datos clínicos apoyan los hallazgos preclínicos que dicen que E4 tiene efectos antiestrogénicos sobre las células de cáncer de mama. Un segundo estudio clínico multicéntrico, abierto fase IB/IIA en 12 pacientes con cáncer de mama avanzado y/o metastásico con RE +/HER2 - resistente a los antiestrógenos evaluó la toxicidad, seguridad, tolerabilidad y la eficacia de altas dosis del E4. ${ }^{48}$ Las dosis de 20, 40 o 60 mg al día de E4 durante 12 semanas fueron bien toleradas, sin observar eventos adversos relacionados al tratamiento. Cinco de nueve pacientes que completaron 12 semanas de tratamiento mostraron efectos antitumorales.

En conjunto, estos resultados destacan que E4 tiene un limitado impacto en el cáncer de mama y puede ofrecer una ventana terapéutica más segura para el tratamiento de los síntomas de la menopausia.

\section{CONCLUSIONES}

El estetrol (E4), estrógeno natural con actividad farmacológica particular (Native Estrogen with Selective action in Tissues $\mathrm{NEST}^{\circledR}$ ) se caracteriza por:

1. Tener menor interferencia con la función hepática, en especial con los parámetros hemostáticos y posiblemente menor impacto en fenómenos trombóticos.

2. Elevada biodisponibilidad oral y larga vida media, lo que habilita una toma diaria.

3. Menor incidencia de enfermedad hepatobiliar.

4. Ausencia de inhibición del citocromo P450 a nivel hepático con menores interacciones medicamentosas con los fármacos que se metabolizan por esta vía.

5. Efecto antitumoral sobre la mama en presencia de estradiol.

6. E4 no tiene metabolitos activos en contraste con E2, cuyo metabolismo conduce a la producción de catecolestrógenos considerados carcinogénicos. ${ }^{49}$

De manera adicional, en términos de seguridad farmacológica, las dosis más bajas de E4 parecen estar asociadas a un menor impacto sobre los lípidos séricos, y una estimulación limitada del tejido mamario. La potencia de la dosis de $10 \mathrm{mg}$ de $\mathrm{E} 4 \mathrm{a}$ nivel endometrial parece ser similar al de la dosis de 2 mg de ValE2, por esta razón, las dosis más altas de E4 utilizadas y estudiadas ( $\geq 15 \mathrm{mg}$ ) en los AOC con E4 pueden garantizar un control del ciclo más satisfactorio en comparación con los AOC basados en E2 (es decir, baja tasa de sangrado y/o manchado y ausencia de sangrado). Esta tercera generación estrogénica de AOC podría proporcionar más beneficios para la salud de la mujer y en los próximos años será un tema de gran interés y mayor personalización en la tecnología anticonceptiva hormonal. 
En la terapia hormonal de la menopausia, E4 parece estar desprovisto de actividad trombogénica, conservando los efectos beneficiosos de E2 a nivel cardiovascular, además de bajo impacto sobre proliferación del tejido mamario. Por lo anterior puede revelarse como un estrógeno innovador y más seguro.

\section{REFERENCIAS}

1. Diczfalusy E. Endocrine functions of the human fetoplacental unit. Fed Proc. 1964; 23: 791-798. Reimpreso en: Am J Obstet Gynecol. 2005; 193 (6): 2024; discussion 2025.

2. Zucconi G, Lisboa BP, Simonitsch E, Roth L, Hagen AA, Diczfalusy E. Isolation of 15-alpha-hydroxy-oestriol from pregnancy urine and from the urine of newborn infants. Acta Endocrinol (Copenh). 1967; 56 (3): 413-423. doi: 10.1530/acta.0.0560413.

3. Holinka CF, Diczfalusy E, Coelingh Bennink HJ. Estetrol: a unique steroid in human pregnancy. J Steroid Biochem Mol Biol. 2008; 110 (1-2): 138-143. doi: 10.1016/j.jsbmb.2008.03.027.

4. Visser M, Holinka CF, Coelingh Bennink HJ. First human exposure to exogenous single-dose oral estetrol in early postmenopausal women. Climacteric. 2008; 11 Suppl 1: 31-40. doi: 10.1080/13697130802056511.

5. Kundu N, Carmody PJ, Didolkar SM, Petersen LP. Sequential determination of serum human placental lactogen, estriol, and estetrol for assessment of fetal morbidity. Obstet Gynecol. 1978; 52 (5): 513-520.

6. Kunzig HJ, Rolleke M, Schmitz-Rockerath B, Dean PG. Value of estetrol determinations in the management of intrauterine growth retardation. Comparison with free estriol. J Perinat Med. 1981; 9 (6): 302-310. doi: 10.1515/jpme.1981.9.6.302.

7. Tulchinsky D, Frigoletto FD Jr, Ryan KJ, Fishman J. Plasma estetrol as an index of fetal well-being. J Clin Endocrinol Metab. 1975; 40 (4): 560-567. doi: 10.1210/jcem-40-4-560.

8. Sciarra JJ, Tagatz GE, Notation AD, Depp R. Estriol and estetrol in amniotic fluid. Am J Obstet Gynecol. 1974; 118 (5): 626-642. doi: 10.1016/s0002-9378(16)33738-3.

9. Notation AD, Tagatz GE. Unconjugated estriol and 15alphahydroxyestriol in complicated pregnancies. Am J Obstet Gynecol. 1977; 128 (7): 747-756. doi: 10.1016/0002-9378(77)90715-3.

10. Martucci C, Fishman J. Uterine estrogen receptor binding of catecholestrogens and of estetrol $(1,3,5(10)$-estratriene3,15alpha,16alpha,17beta-tetrol). Steroids. 1976; 27 (3): 325-333. doi: 10.1016/0039-128x(76)90054-4.

11. Tseng L, Gurpide E. Competition of estetrol and ethynylestradiol with estradiol for nuclear binding in human endometrium. J Steroid Biochem. 1976; 7 (10): 817-822. doi: 10.1016/00224731(76)90184-9.

12. Jozan S, Kreitmann B, Bayard F. Different effects of oestradiol, oestriol, oestetrol and of oestrone on human breast cancer cells (MCF-7) in long term tissue culture. Acta Endocrinol (Copenh). 1981; 98 (1): 73-80. doi: 10.1530/acta.0.0980073.

13. Holinka CF, Gurpide E. In vivo effects of estetrol on the immature rat uterus. Biol Reprod. 1979; 20 (2): 242-246. doi: 10.1093/ biolreprod/20.2.242.

14. Holinka CF, Bressler RS, Zehr DR, Gurpide E. Comparison of effects of estetrol and tamoxifen with those of estriol and estradiol on the immature rat uterus. Biol Reprod. 1980; 22: 913-926. doi: 10.1095/ biolreprod22.4.913.

15. Coelingh Bennink HJ, Holinka CF, Diczfalusy E. Estetrol review: profile and potential clinical applications. Climacteric. 2008; 11 Suppl 1: 47-58. doi: 10.1080/13697130802073425.
16. Warmerdam EG, Visser M, Coelingh Bennink HJ, Groen M. A new route of synthesis of estetrol. Climacteric. 2008; 11 Suppl 1: 59-63. doi: 10.1080/13697130802054078.

17. Visser M, Foidart JM, Coelingh Bennink HJT. In vitro effects of estetrol on receptor binding, drug targets and human liver cell metabolism. Climacteric. 2008; 11 (Suppl 1): 64-68. doi: 10.1080/13697130802050340.

18. Coelingh Bennink HJT, Singer C, Simoncini T, Genazzani AR, Holinka CF, Kubista E. Estetrol, a pregnancy-specific human steroid, prevents and suppresses mammary tumor growth in a rat model. Climacteric. 2008; 11 (Suppl 1): 29. doi: 10.1080/13697130802040325.

19. Coelingh Bennink HJT, Heegaard AM, Visser M, Holinka CF, Christiansen C. Oral bioavailability and bone-sparing effects of estetrol in an osteoporosis model. Climacteric. 2008; 11 (Suppl 1): 2-14. doi: 10.1080/13697130701798692.

20. Hammond GL, Hogeveen KN, Visser M, Coelingh Bennink HJT. Estetrol does not bind sex hormone binding globulin or increase its production by human HepG2 cells. Climacteric. 2008; 11 (Suppl 1): 41-46. doi: 10.1080/13697130701851814.

21. Heegaard AM, Holinka CF, Kenemans P, Coelingh Bennink HJT. Estrogenic uterovaginal effects of oral estetrol in the modified Allen-Doisy test. Climacteric. 2008; 11 (Suppl 1): 22-28. doi: 10.1080/13697130701842490.

22. Holinka CF, Brincat M, Coelingh Bennink HJT. Preventive effect of oral estetrol in a menopausal hot flush model. Climacteric. 2008; 11 (Suppl 1): 15-21. doi: 10.1080/13697130701822807.

23. Coelingh Bennink HJT, Skouby S, Bouchard P, Holinka CF. Ovulation inhibition by estetrol in an in vivo model. Contraception. 2008; 77: 186-190. doi: 10.1016/j.contraception.2007.11.014.

24. Estetrol (E4), the first NEST ${ }^{\top M}$. [Access 16-02-2021] Available in: https://www.mithra.com/en/estetrol

25. Coelingh Bennink HJT, Verhoeven C, Zimmerman Y, Visser M, Foidart JM, Gemzell-Danielson K. Pharmacodynamic effects of the fetal estrogen estetrol in postmenopausal women: results from a multiplerising-dose study. Menopause. 2017; 24: 677-685. doi: 10.1097/ GME.0000000000000823.

26. Coelingh Bennink HJT, Verhoeven C, Zimmerman Y, Visser M, Foidart JM, Gemzell-Danielson K. Pharmacokinetics of the fetal estrogen estetrol in a multiple-rising-dose study in postmenopausal women. Climacteric. 2017; 20 (3): 285-289. doi: 10.1080/13697137.2017.1291608.

27. Grandi G, Facchinetti F, Bitzer J. Estradiol in hormonal contraception: real evolution or just same old wine in a new bottle? Eur J Contracept Reprod Health Care. 2017; 22 (4): 245-254. doi: 10.1080/13625187.2017.1372571.

28. Fishman J. Fate of $15 \mathrm{a}$-hydroxyestriol-3H in adult man. J Clin Endocrinol. 1970; 31: 436-438. doi: 10.1210/jcem-31-4-436.

29. Fishman J, Schut H, Solomon S. Metabolism, production and excretion rates of 15a-hydroxyestriol in late pregnancy. J Clin Endocrinol Metab. 1972; 35: 339-344. doi: 10.1210/jcem-35-3-339.

30. Duijkers IJM, Klipping C, Zimmerman Y, Appels N, Jost M, Maillard $C$ et al. Inhibition of ovulation by administration of estetrol in combination with drospirenone or levonorgestrel: results of a phase II dose-finding pilot study. Eur J Contracept Reprod Health Care. 2015; 20: 476-489. doi: 10.3109/13625187.2015.1074675.

31. Apter D, Zimmerman Y, Beekman L, Mawet M, Maillard C, Foidart JM et al. Bleeding pattern and cycle control with estetrol-containing combined oral contraceptives: results from a phase II, randomised, dose-finding study (FIESTA). Contraception. 2016; 94 (4): 366-373. doi: 10.1016/j.contraception.2016.04.015.

32. Apter D, Zimmerman Y, Beekman L, Mawet M, Maillard C, Foidart JM et al. Estetrol combined with drospirenone: an oral contraceptive with high acceptability, user satisfaction, well-being and favourable 
body weight control. Eur J Contracept Reprod Health Care. 2017; 22 (4): 260-267. doi: 10.1080/13625187.2017.1336532.

33. Mawet M, Maillard C, Klipping C, Zimmerman Y, Foidart JM, Coelingh Bennink HJ. Unique effects on hepatic function, lipid metabolism, bone and growth endocrine parameters of estetrol in combined oral contraceptives. Eur J Contracept Reprod Health Care. 2015; 20 (6): 463-475. doi: 10.3109/13625187.2015.1068934.

34. Klipping C, Duijkers I, Mawet M, Maillard C, Bastidas A, Jost M et al. Endocrine and metabolic effects of an oral contraceptive containing estetrol and drospirenone. Contraception. 2021; 103 (4): 213-221. doi: 10.1016/j.contraception.2021.01.001.

35. Dragoman MV, Tepper NK, Fu R, Curtis KM, Chou R, Gaffield ME. A systematic review and meta-analysis of venous thrombosis risk among users of combined oral contraception. Int J Gynaecol Obstet. 2018; 141 (3): 287-294. doi: 10.1002/ijgo.12455.

36. Archer D, Ahrendt HJ, Drouin D. Drospirenone-only oral contraceptive: results from a multicenter noncomparative trial of efficacy, safety and tolerability. Contraception. 2015; 92 (5): 439-444. doi: 10.1016/j.contraception.2015.07.014.

37. Medical eligibility criteria for contraceptive use. Progestogen-only contraceptives. Geneva: World Health Organization. 2015. p. 157.

38. Kluft C, Zimmerman Y, Mawet M, Klipping C, Duijkers IJM, Neuteboom $\mathrm{J}$ et al. Reduced hemostatic effects with drospirenone-based oral contraceptives containing estetrol vs. ethinyl estradiol. Contraception. 2017; 95 (2): 140-147. doi: 10.1016/j.contraception.2016.08.018.

39. Douxfils J, Klipping C, Duijkers I, Kinet V, Mawet M, Maillard C et al. Evaluation of the effect of a new oral contraceptive containing estetrol and drospirenone on hemostasis parameters. Contraception. 2020; 102 (6): 396-402. doi: 10.1016/j.contraception.2020.08.015.

40. Coelingh Bennink HJT, Verhoevena C, Zimmerman Y, Vissera $M$, Foidart JM, Gemzell-Danielsson K. Clinical effects of the fetal estrogen estetrol in a multiple-rising-dose study in postmenopausal women. Maturitas. 2016; 91: 93-100. doi: 10.1016/j.maturitas.2016.06.017.

41. Gaspard U, Taziaux M, Mawet M, Jost M, Gordenne V, Coelingh Bennink HJT et al. A multicenter, randomized study to select the minimum effective dose of estetrol (E4) in postmenopausal women (E4Relief): part 1. Vasomotor symptoms and overall safety. Menopause. 2020; 27 (8): 848-857. doi: 10.1097/GME.0000000000001561.

42. Arnal JF, Lenfant F, Metivier R, Flouriot G, Henrion D, Adlanmerini M et al. Membrane and nuclear estrogen receptor alpha actions: from tissue specificity to medical implications. Physiol Rev. 2017; 97 (3): 1045-1087. doi: 10.1152/physrev.00024.2016.

43. Abot A, Fontaine C, Buscato M, Solinhac R, Flouriot G, Fabre A et al. The uterine and vascular actions of estetrol delineate a distinctive profile of estrogen receptor $\alpha$ modulation, uncoupling nuclear and membrane activation. EMBO Mol Med. 2014; 6 (10): 1328-1346. doi: 10.15252/emmm.201404112.

44. Gérard C, Mestdagt M, Tskitishvili E, Communal L, Gompel A, Silva E et al. Combined estrogenic and anti-estrogenic properties of estetrol on breast cancer may provide a safe therapeutic window for the treatment of menopausal symptoms. Oncotarget. 2015; 6 (19): 17621-1736. doi: 10.18632/oncotarget.4184.

45. Giretti MS, Montt Guevara MM, Cecchi E, Mannella P, Palla G, Spina $S$ et al. Effects of estetrol on migration and invasion in T47-D breast cancer cells through the actin cytoskeleton. Front Endocrinol (Lausanne). 2014; 5: 80. doi: 10.3389/fendo.2014.00080.

46. Visser M, Kloosterboer HJ, Bennink HJ. Estetrol prevents and suppresses mammary tumors induced by DMBA in a rat model. Horm Mol Biol Clin Investig. 2012; 9 (1): 95-103. doi: 10.1515/ hmbci-2012-0015.

47. Singer CF, Bennink HJ, Natter C, Steurer S, Rudas M, Moinfar F et al. Antiestrogenic effects of the fetal estrogen estetrol in women with estrogen-receptor positive early breast cancer. Carcinogenesis. 2014; 35 (11): 2447-2451. doi: 10.1093/carcin/bgu144.

48. Schmidt M, Lenhard H, Hoenig A, Zimmerman Y, Krijgh J, Jansen M et al. Tumor suppression, dose-limiting toxicity and wellbeing with the fetal estrogen estetrol in patients with advanced breast cancer. J Cancer Res Clin Oncol. 2021; 147 (6): 1833-1842. doi: 10.1007/ s00432-020-03472-8.

49. Mauras N, Torres-Santiago L, Santen R, Mericq V, Ross J, Colon-Otero $\mathrm{G}$ et al. Impact of route of administration on genotoxic oestrogens concentrations using oral vs transdermal oestradiol in girls with Turner syndrome. Clin Endocrinol (Oxf). 2019; 90 (1): 155-161. doi: 10.1111/cen.13869.

Conflicto de intereses: Teresa Navarrete Horta, Francisco Guerrero Carreño y Paulina Valdés Aguerrebere declaran que no tienen ningún conflicto de intereses. Leopoldo Alejandro Vázquez Estrada es Asesor Médico Científico de Gedeon Richter México. 